\title{
A BRIEF HISTORY OF OUR PERCEPTION OF THE SOLAR TACHOCLINE
}

\author{
D. Gough ${ }^{1}$
}

\begin{abstract}
I tell just part of the story of the quest to understand the dynamics of the solar tachocline, from the point of view of my relationship with my good friend Jean-Paul Zahn.
\end{abstract}

\section{Prelude}

By having a common scientific interest, namely astrophysical fluid dynamics, it was natural that Jean-Paul and I would become friends. I first met Jean-Paul in the summer of 1968 participating in the Program of Geophysical Fluid Dynamics (GFD) held in Walsh College (as it was affectionately known) at the Woods Hole Oceanographic Institution. We immediately got on very well indeed, although I hasten to add that that was principally due to Jean-Paul, who is so friendly and kind that he gets on well with everyone whom he encounters. After that meeting we interacted a great deal over the years, but more about that later. What I have been asked by the organizers of this meeting to do here is to provide a discussion of an aspect of the subject with which both he and I have been deeply concerned, namely the solar tachocline. I shall not give a balanced account of the subject as a whole; rather, I shall concentrate on my own view of the development of our separate ideas, particularly where they have overlapped.

\section{Background}

It was Easter Sunday 1965. I was in the library of the Department of Applied Mathematics and Theoretical Physics in Cambridge, where I was a research student, reading two papers by Lord Rayleigh on instabilities in rotating fluids. In the first is derived what is now called the Rayleigh condition: a uniform incompressible inviscid flow, circularly swirling, is stable to axisymmetric perturbations

${ }^{1}$ Department of Applied Mathematics and Theoretical Physics\& Institute of Astronomy, Madingley Road, Cambridge, CB3 0HA, UK 
if the magnitude of vorticity increases outwards everywhere (I paraphrase); the second paper, which is predominantly devoted to rectilinear shear flow, reports that a necessary condition for instability to two-dimensional perturbations is that the vorticity has a turning point somewhere (again I paraphrase; as Rayleigh has pointed out, the criterion generalizes to swirling flow - later, it transpired that in the criterion, which is commonly somewhat misleadingly called the inflexionpoint condition, the magnitude of the vorticity has to be a maximum; and, more generally, it appears that vorticity should be replaced by potential vorticity, by which I mean $\omega \cdot \nabla s / \rho$, where $\omega$ is vorticity, $s$ is specific entropy and $\rho$ is density.) What interested me was that although incomplete, the two criteria both involved (potential) vorticity alone. As I was pondering the matter, in walked Donald Lynden-Bell. He was at a loose end because his wife was in hospital about to give birth to their first child, and he had come into the department to shelter from the snow - not typical weather in Cambridge, especially at Easter. I was there because my wife was in the same hospital, on duty as a nurse. It turned out that Donald had been thinking along the same lines, but more thoroughly (and more deeply) than I, and was in the process of generalizing the results to inhomogeneous fluids that were either not in pure rotation or not purely rectilinear. We talked for the rest of the day, and came to the conclusion first that any turbulence resulting from such instabilities, especially if the dominant energy-containing eddies are almost two-dimensional, produces Reynolds stresses that would, in the first instance, tend not to smooth out large-scale velocity, as was commonly assumed, but more likely large-scale vorticity instead. We conjectured that turbulence caused by, for example, convection, would behave similarly, and later carried out an experiment which we though (mistakenly) demonstrated the process. Our conclusion was not entirely new: we subsequently learned that G I Taylor $(1915,1932)$ had come to a similar conclusion some decades before, and had developed his mixing-length ideas based on vorticity conservation. Moreover, Richard Scorer, an eminent meteorologist at Imperial College, in an unpublished report had also come to the conclusion that turbulence tends to homogenize vorticity, although his view of the large-scale consequences were different from ours. Nevertheless, it convinced us both that turbulent fluids are unlikely to rotate uniformly. Therefore convection zones in stars would rotate differentially, as was known to be the case in the Sun. Our conjecture was surely inaccurate in detail, and it subsequently led me into future interesting debates with Jean-Paul.

A similar conclusion might have been drawn of radiative zones, but for a different reason. I learned later that year from Francis Bretherton, the principal lecturer in the 1965 GFD programme, that, in sheared stably stratified regions, internal gravity waves (which in stars are likely to be generated at the interface between convective and radiative zones) dissipate asymmetrically in such a way as to enhance, rather than to reduce, the shear. Francis's emphasis was on wave absorption near critical layers ( $c f$. Booker \& Bretherton 1967), but it was clear that shear enhancement would occur elsewhere too, albeit very much more weakly $(c f$. Eliassen \& Palm 1960). Therefore for this reason alone in the absence of a magnetic field a radiative zone in any star is likely not to rotate uniformly, although 
other processes, such as advection by Eddington-Sweet circulation, might dominate the tendency towards nonuniformity. However, Maxwell stresses can act in such a way as to rigidify the star.

\section{New York}

It was with these ill-formed ideas that I went to New York to work on a model of convection with Ed Spiegel and Jüri Toomre. Jüri has already discussed what we $\operatorname{did}^{1}$, but I mention it here simply to note that as I was leaving to return to Cambridge Jean-Paul arrived in New York to help extend our work to study the nonlinear development of instabilities in rectilinear shear flow. I interacted as much as I could, but before the days of internet and Skype that was an inefficient process. So, aside from working on the formulation of the model equations, my subsequent contribution to that endeavour was minimal. However, what interaction there was strengthened my friendship with Jean-Paul, and led me to realize that his intellect matched up to his enormous kindness. Little was discussed about rotating flows at that time, although soon after we both wrote independent reviews on the subject in conference proceedings (Zahn 1974; Gough 1976). We both had an interest in angular-momentum transport by gravity waves, and we resolved to get together to work jointly on the subject.

\section{Nice}

Jean-Paul left New York to take up the Directorship of the Observatoire de Nice. I visited him briefly at the Observatoire on a couple of occasions, and then in 1977 he arranged for me spend my first sabbatical year there. It was a wonderful year for my whole family in a most delightful part of the world. Before arriving we had worried that tourists might overwhelm us, but we quickly discovered that they confine themselves to a half-kilometre-wide strip along the coast, so it was quite simple to avoid even the awareness of their presence. Jean-Paul was, of course, an impeccable host, and we spent many pleasant times with him and his family. One memorable occasion was when we were all invited to dinner at their house after the Zahns had returned from a holiday in Corsica. As part of the cheese course my wife Rosanne, usually a very gentile lady, as Jean-Paul used to describe her, was offered a greyish-coloured soft cheese which Jean-Paul recommended very highly as being exciting and most typically Corsican. When Rosanne sampled it, with Jean-Paul regarding her expectantly, her whole mouth instantly dried up completely; she was quite unable to utter a word for several minutes, although her gesticulations made it quite clear what she might have said had she been able. Jean-Paul was now roaring with laughter in his characteristically contagious way: so infectious was it that even Rosanne joined in with the rest of us once she was able; Jean-Paul took great delight in recalling that story on many occasions later.

\footnotetext{
${ }^{1}$ Editor's note: see J. Toomre's contribution these proceedings.
} 
Jean-Paul was a very conscientious director, giving his time to all who called upon him. I learned a great deal from observing his techniques, and years later tried to emulate them when I was Director of the Institute of Astronomy in Cambridge, although never with such aplomb as Jean-Paul. One of Jean-Paul's great skills was to circumnavigate the obstructions laid before him by the French bureaucratic system, showing at least on paper that he had obeyed the rules scrupulously, and at the same time operating with complete integrity: "honesty in the large", he called it. It maximized efficiency and satisfaction. It taught me that the principal duty of a director of an academic institution is to insulate the staff from outside bureaucratic interference so that they can devote their time more profitably to scholarship and research.

One of the consequences of Jean-Paul's selfless style was that the freedom he gave to his staff for getting on with their work almost unhindered had to be paid for with his own time. That hindered his own research. During my sabbatical leave, Jean-Paul would set aside periods free from prior appointments in order to spend full mornings for research with me in my office, in a building far distant from the administrative wing of the observatory. Ostensibly, only his secretary knew his whereabouts, and was to phone only in the case of the most dire emergency. That happened typically about 20 to 30 minutes into each visit; Jean-Paul would excuse himself for "un petit quart d'heure" 2 , but that was the last I would see of him: Jean-Paul was so kind to his staff that he would always sacrifice his precious time to their whim. So, sadly, we did not make any joint progress on gravity-wave transport in stars. We did, however, find some time for all-too-brief discussions about solar spin-down, the role of angular-momentum redistribution in stellar convection zones, and the structure of the putative solar tachocline which Ed Spiegel (1972) had adumbrated. Our deliberations were most useful to me, because Jean-Paul had a deep physical understanding of fluid dynamics, although our opinions about the angular-velocity distribution in rotating turbulent flows continued to differ, maintaining a healthy arena for debate.

During most of the rest of my stay in Nice I worked on developing helioseismological inferencing procedures, in part with other members of the observatory, Gabriel Berthomieu, Janine Provost and Arlette Rocca, together with my student from Cambridge, Alan Cooper, and also Yoji Osaki, who was also visiting on sabbatical leave. Subsequently, in the fullness of time, seismic observations became good enough to reveal the angular velocity $\Omega$ throughout most of the Sun: broadly speaking, the latitudinal variation observed at the surface persists essentially to the base of the convection zone, and the radiative envelope beneath rotates roughly uniformly, the two regimes being separated by the tachocline (Brown et al. 1989; Goode et al. 1991; Sekii 1991; Thompson et al. 1996), a shear layer too thin to be resolved and whose presence was no doubt closely related to Ed Spiegel's

2 This is meant to mean a "brief" interval of time, although what "brief" actually means depends on the location in France in which it is used; in my experience, in the Côte d'Azur it is typically between an hour and a half and an hour and three-quarters, although I believe that Jean-Paul meant it literally. 


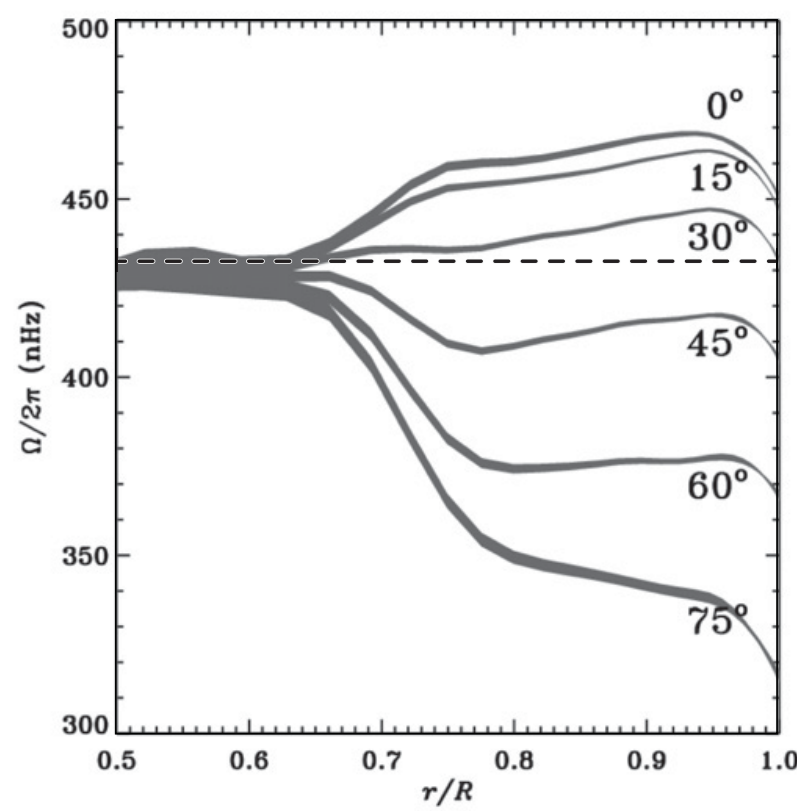

Fig. 1. Seismologically determined rotation rate of the solar interior, plotted against fractional radius at various latitudes, taken from the US National Solar Observatory's archives. The horizontal dashed line denotes the cyclic frequency $434.6 \mathrm{nHz}$.

prediction. The angular velocity $\Omega_{0}$ in the radiative interior is such that the spherically averaged angular momentum density is more-or-less continuous across the tachocline, which translates to the value of $\Omega$ in the convection zone at a latitude of about $30^{\circ}$. The variation of $\Omega$ is illustrated in Figure 1. Two obvious questions naturally arise: why does the radiative interior rotate uniformly, and what determines the rate? Additionally, what determines the tachocline thickness?

\section{Les Houches}

A decade later Jean-Paul, together with Jean Zinn-Justin, organized a summer school in Les Houches on Astrophysical Fluid Dynamics. It was a great success, both academically and, of course, socially, and many of the students at the time have since become senior academics. I was one of the lecturers - on stellar oscillations, not stellar rotation - yet Jean-Paul and I, and also Ed Spiegel, did find time to discuss rotational dynamics. My wife, Rosanne, and I, and our son Russell, were housed, by choice, in a somewhat dilapidated old cabin at the edge of a wood, away from the main school premises, in preference to the lecturers' modern apartment block which had no character. One day, as I was preparing my next lecture, I was overcome by an excruciating pain in my back, and I fell to the 
floor. Rosanne passed by, asked if I was OK (to which I replied "no", which I'm sure she didn't hear), and continued on her way. Half an hour later she passed by again, and chided me for staying on the dirty floor. Then my plight became clear, and Jean-Paul's assistance was summoned. On his arrival, once he had established that I wasn't in mortal danger, Jean-Paul burst into his characteristic laughter. Then he showed his usual kindness, and called for a doctor from nearby Chamonix. It was not until well after lunchtime that the doctor arrived, reeking of alcohol; he hardly even entered the room, declared lumbago, prescribed some injections to be administered by Rosanne, and promptly left, after the settling of an exorbitant account. Jean-Paul saw the funny side of the encounter, of course, but deep down showed his true sympathy; he telephoned his son, Pierre-Yves, a physiotherapist near Toulouse, who assured us that what had been prescribed was simply a muscle relaxant, and, whatever my ailment really was, it would at least do no harm. Jean-Paul then had to reschedule lectures, postponing the rest of my course for a couple of weeks. There were many walks in the mountains to be enjoyed, in which, sadly, I could no longer participate. On one occasion, Jean-Paul kindly took Russell, my eight-year-old son, who was full of energy and often ran on ahead. Russell took a wrong fork in the path, and before either he or Jean-Paul realized it, he had descended into the wrong valley. There was panic on both sides, I learned subsequently, Russell fearing the consequences of being lost and Jean-Paul fearing the consequences of having lost him. It was quite a long time before a happy reunion ensued. Neither Jean-Paul nor Russell ever forgot that experience, which, quite naturally, led to a life-long bond between them.

\section{Spiegel-Zahn tachocline dynamics}

The biggest single step towards understanding the tachocline was made in a seminal paper by Ed and Jean-Paul in 1992. They accepted that the Reynolds stresses and the associated meridional circulation dominate the dynamics in the convection zone, and are essentially uninfluenced by any motion beneath. They then enquired of the reaction of a purely radiative, initially uniformly rotating, interior abutting the differentially rotating fluid above. Their first quest was in addressing this question subject to the assumption that the only momentum or energy transporting agent other than advection is radiative diffusion. Assuming axisymmetry, they found that a thermally induced circulation was driven by the convection-zone shear, which burrowed deeply through the radiative envelope resulting, over the main-sequence life of the Sun, in nonuniformity in the angular velocity right down to the energy-generating core, leaving no boundary layer at the interface with the convection zone. Therefore some other angular-momentum transporting agent is essential for explaining the helioseismological findings. The outcome must involve the production of the tachocline.

Ed and Jean-Paul recalled the Ekman layer which was predicted to develop in solar spin-down (Spiegel 1972), and mimicked it by positing that the tachocline is turbulent, apparently as a result of shear instability, the hindrance of vertical motion by the stable stratification greatly reducing the transport of angular 
momentum vertically relative to the transport horizontally. The turbulence was implicitly assumed to be horizontally isotropic, and its transport properties were modelled by a viscous stress, the coefficient of viscosity being much larger when multiplying the horizontal shear than the vertical. The outcome was that the stress opposing horizontal shear leads to uniform rotation sufficiently far beneath the base of the convection zone, which the relatively weak vertical stress is unable to disturb. Thus the lower reaches of the tachocline act somewhat like the rigid boundary in an typical (terrestrial) spin-down situation, and the dynamics in the bulk of the tachocline resembles that in an Ekman boundary layer. Because the vertical viscous stress in small, the tachocline is thin, and the dynamics within it is not severely influenced by the stratification. The horizontal Ekman flow at low latitudes within the tachocline, where the angular velocity exceeds that of the deep interior, is towards the axis of rotation, just as in the boundary layer at the bottom of a stirred cup of tea during spin-down (e.g. Greenspan 1969) ${ }^{3}$; at high latitudes the flow is in the opposite direction. The opposing flows converge at the latitude of no vertical shear, which, when the turbulent viscosity is presumed to be independent of latitude, was found to be "approximately" $42^{\circ}$ (actually closer to $43^{\circ}$ ). That determines $\Omega_{0}$. Contours of angular velocity, according to this model, are illustrated in Figure 2.

Julian Elliott (1997) subsequently carried out numerical simulations to test the Spiegel-Zahn theory. He reproduced the Ekman flow, converging at a latitude of about $40^{\circ}$, and the interior angular velocity $\Omega_{0}$ of about $0.91 \Omega_{\mathrm{e}}$, where $\Omega_{\mathrm{e}}$ is the equatorial angular velocity in the convection zone. (This is to be compared with the seismologically determined value of about $0.95 \Omega_{\mathrm{e}}$. $)^{4}$ However, to achieve that he required the horizontal component of the turbulent viscosity to be about $5 \times 10^{5} \mathrm{~cm}^{2} \mathrm{~s}^{-1}$, which is significantly smaller than a lower bound estimated earlier by Jean-Paul (Zahn 1992). Nevertheless, it seems to me that, subject to the assumption that the tachocline dynamics is indeed dominated by turbulence, and that the turbulence acts like viscosity, albeit relatively weakly in the vertical, the essence of Ed and Jean-Paul's conclusion is unassailable.

Of course, a different agent might cause the radiative interior to rotate rigidly. For example, Jean-Paul, Suzanne Talon and José Matias (Zahn 1994; Zahn et al. 1997) investigated the possibility that dissipation of internal gravity waves might hold the interior rigid, notwithstanding its general tendency to enhance, rather than suppress, shear. I think it is fair to say that they did not produce a convincing case. Although Tami Rogers (2011) has carried out some very interesting (two-dimensional) simulations suggesting that the interior could be held rigid, I suspect that her computations were too diffusive to apply directly to the Sun. In

\footnotetext{
${ }^{3}$ Near the equator the angular-velocity vector is nearly parallel to the tangent plane of the boundary layer, and not perpendicular as in a cup of tea. However, that does not change the balance of forces in a critical way, as Bondi and Lyttleton (1948) had demonstrated in their analysis of the spin-down of the Earth.

${ }^{4}$ Agreement could, of course, be engineered by invoking an appropriate latitudinal variation of the turbulent viscosity.
} 


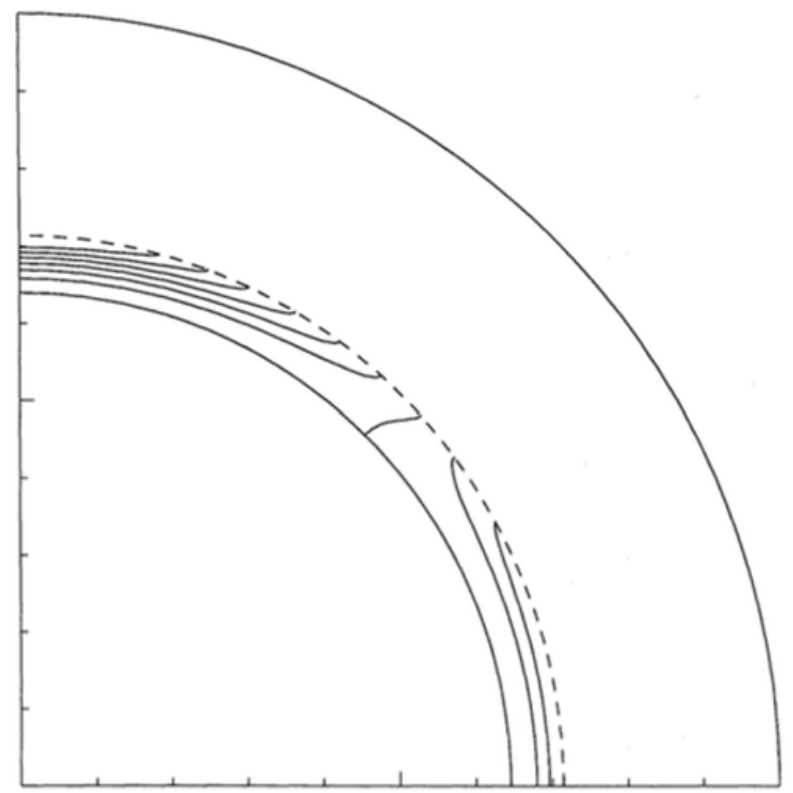

Fig. 2. Angular velocity contours beneath the convection zone (whose base is represented by the dashed curve) according to the Spiegel-Zahn theory. The angular velocity beneath the tachocline, whose thickness has been set arbitrarily to $50 \mathrm{Mm}$, is almost uniform, equal to the value at the base of the convection zone at a latitude of approximately $42^{\circ}$ (from Spiegel \& Zahn 1992).

contrast, others, such as David Fritts, Sharon Vadas \& Oyvind Andreassen (1998) and Eun-Jin Kim and Keith MacGregor (2005), have discussed gravity-wave enhancement of shear in the solar case.

Another possible agent, one that has been discussed often, is a global magnetic field (e.g. Rosner and Weiss 1985; Mestel and Weiss 1987; Charbonneau \& MacGregor 1993; MacGregor \& Charbonneau 1999). Indeed, Rüdiger and Kitchatinov (1997) have pointed out that because magnetic diffusion in the radiative interior is slow in comparison with the timescale of the shear in the convection zone, a simple Hartmann layer at the interface would be thin and so might provide the explanation of the tachocline. However, they provided no cogent explanation of how the differential rotation of the convection zone is not imprinted on the radiative region beneath, but simply decreed what is tantamount to the vanishing of Maxwell stresses at the interface on the ground that the eddy diffusivity in the convection zone is large.

\section{An alternative view of tachocline dynamics}

In view of my belief that layerwise two-dimensional turbulence locally has a stronger tendency to homogenize potential vorticity rather than angular velocity, I could 
not accept the manner in which turbulence was presumed to operate in the SpiegelZahn tachocline. On the whole the nonlinear development of any instability is to act in such a way as to reduce the destabilizing agent, and in swirling flows the distribution of potential vorticity seems to be the dominant factor. Indeed, I subsequently persuaded Pascale Garaud, then my research student, to carry out a weakly nonlinear computation of the reaction to the instability resulting from a latitudinally differentially rotating convectively stable spherical stellar shell to demonstrate that a local maximum in the potential vorticity is smoothed out (Garaud 2001). However, in the solar tachocline there are more than a single destabilizing agent, so it isn't immediately obvious what the consequences might be. Indeed, Tobias, Diamond and Hughes (2007) have produced evidence that the addition of a weak magnetic field tends to annul turbulent transport tendencies, whether frictional or not. Michael McIntyre drew my attention to some work that he had carried out with Peter Haynes and their colleagues (1991) concerning the development of the instability of a simple rectilinear shear, in which it was shown that mere local homogenization of vorticity could not provide the whole story because it does not conserve both energy and momentum; instead, energy has to be removed by waves, which renders the entire process nonlocal. Michael showed me evidence that layerwise two-dimensional turbulence in the stably stratified layers of the Earth's atmosphere does indeed produce regions of uniform potential vorticity, and generates the requisite waves to enable that to occur - he has subsequently exposed many of the details in the astrophysical literature (McIntyre 2003). He and I discussed the implications regarding the Sun's rotation, and concluded that because fluid-dynamical processes alone cannot hold the Sun rigid, there must necessarily be a large-scale magnetic field pervading the entire radiative envelope. Like Ed and Jean-Paul, we accepted that the dynamics of the convection zone was hardly influenced by the relatively weak flow beneath, and addressed the dynamics of the tachocline separating the two regimes. As in the Spiegel-Zahn view of the dynamics, gyroscopic pumping (e.g. Pedlosky 1987) causes fluid to descend from the convection zone at both high and low latitudes, subsequently to be diverted horizontally in a thin boundary layer by the magnetic field, which it prevents from diffusing outwards almost everywhere, thereby obviating a strong magnetic communication of angular momentum. The field confinement is an essential requirement of the theory, as was recognized also by MacGregor and Charbonneau (1999), for otherwise field distortion by the shear in the convection zone, ignored in several other studies (e.g. Forgács-Dajka \& Petrovay 2001, 2002; Kitchatinov \& Rüdiger 2006), would be imprinted on the radiative interior. Only in the vicinity of convergence does the fluid return to the convection zone, possibly dragging field with it at latitudes where the angular velocities of the convection zone and the radiative interior coincide. Figure 3 is a cartoon of a possible magnetic-field configuration and the meridional tachocline flow.

Michael and I (GM98) presented what is essentially a scale analysis, which relates the mean strength of the magnetic field $B_{0}$ in the boundary layer to the thickness $\Delta$ of the tachocline. Ventilation of the tachocline by the meridional flow has mixed helium that has settled during the Sun's main-sequence evolution back 


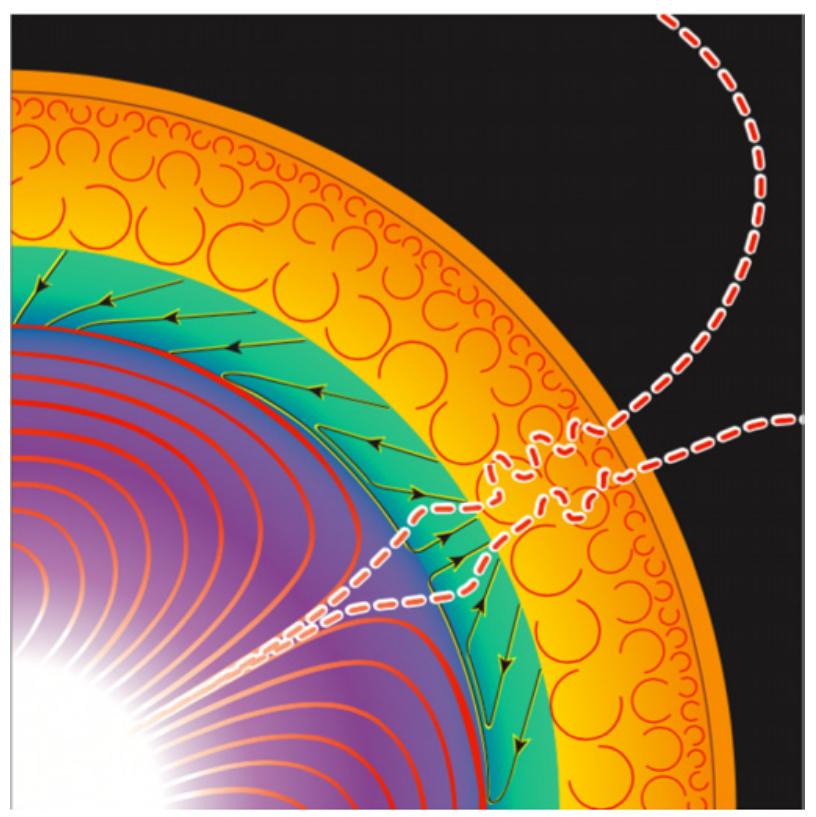

Fig. 3. Flow lines (with arrows) in the tachocline (drawn artificially thick for clarity) and magnetic field (full) lines in the radiative envelope in the style of GM98, but with the dipolar axis inclined to the latitude of zero vertical shear. The dashed field lines are particularly speculative. The circle segments represent turbulent convective cells.

into the convection zone, rendering the tachocline hydrogen-rich relative to standard solar models. A helioseismological calibration of the resulting sound-speed anomaly (Elliott \& Gough 1998) implied that $\Delta \simeq 2 \times 10^{-2} R_{\odot}$, whence $B_{0} \simeq 1 \mathrm{G}$, suggesting a strength of the large-scale interior field of about $10^{3} \mathrm{G}$. In our scale analysis we assumed, solely for simplicity, that the magnetic field is dipolar, the dominant residual of the primeval state (e.g. Braitewaite \& Spruit 2004), with its axis aligned with the axis of rotation. We were confident that our conclusions were valid, except, possibly, near the magnetic poles where the field is nearly vertical, and where the conditions for the validity break down. Nevertheless, we conjectured that in any case the field would still be contained beneath the tachocline, although burrowing of the meridional flow into the radiative interior would be more extensive, resulting in a polar pit which might have implications for lithium burning in the early main-sequence evolution. In that respect we overlooked the fact that the requirement of having to balance the resulting horizontal pressure gradient by Maxwell stresses limits the possible deviation from spherical symmetry to an extent that the influence on lithium burning is unlikely to be significant. Subsequently, Toby Wood and Michael (2011) published a careful model analysis of the polar regions demonstrating that magnetic confinement can still take place. 
It should be realized that the analyses ignored the effect of relatively fast processes, such as magnetorotational instability, which could make a serious quantitative impact on the balance of forces. Several interesting pertinent studies of such processes have been published since the early work (e.g. Kim \& MacGregor 2003; Kim 2005; Diamond et al. 2007) whose implications deserve further investigation. Nevertheless, it seems likely, at least to me, that the qualitative behaviour must persist.

The discussions by both Ed and Jean-Paul, and Michael and me, assumed that the tachocline is essentially stationary. However, there is seismological evidence that the rotational shear in the tachocline is oscillating, or at least has oscillated, with a period of about 1.3 years (Howe et al. 2000). What drives that oscillation? One possibility is the differential absorption of gravity waves generated at the interface with the convection zone acting in the manner of the quasi-biennial oscillation of the Earth's atmosphere (Holton \& Lindzen 1972; Plumb \& McEwan 1978). Indeed, I once contemplated the process as being responsible for the solar cycle (Gough 1998), although I doubted that waves of sufficiently high amplitude to produce so short an oscillation period could be generated. The idea was embraced briefly by Jean-Paul (Kumar et al. 1999), who estimated an enormously greater gravity-wave amplitude for waves that penetrate more deeply. However, there remains the possibility that the magnetic field provides the principal restoring force, and gravity-wave dissipation provides the driving. The oscillation could also induce meridional flow (Fritts, Vadas \& Andreassen 1998), which might conceivably provide a significant transport mechanism for chemical species, and possibly explain the Li deficiency in the Sun's photosphere.

\section{Numerical simulations}

It is clear that tachocline dynamics is highly nonlinear, so it has been natural to revert to numerical simulation. Plausible conclusions were evidently not easy to come by, because even the simplest of models have nested boundary layers which require a hierarchy of small diffusion coefficients for adequate modelling, thereby demanding some of them to be so small as to present severe numerical challenges. I recall one summer sharing some time with Jean-Paul and Jüri Toomre at JILA in Boulder, Colorado, during which we discussed how such coefficients could in practice be chosen for a potentially useful three-dimensional computation: it was less than a decade after Les Houches, and computers were still woefully inadequate to cope with even half-realistic values.

One weekend during our sojourn, kindly made possible by Jüri, Rosanne and I and our two sons, Julian and Russell, went camping in the Rocky Mountains. It was early Sunday morning. As we were brewing coffee for breakfast, we heard an unmistakable laugh. I rushed off towards the source of the sound, and quite soon encountered Jean-Paul who was looking for us; he had been aware of only the broad area within which we might be found. In his arms was a large bag of freshly baked croissants, to provide us with a breakfast the kind of which is never 
dreamt of in a wilderness. Jean-Paul's arrival was certainly a surprise, but was quite typical of the many unusual kind antics that he enjoyed so much.

One of the conundrums to be faced by a theory of the tachocline is posed by the manner in which the magnetic field in the radiative interior can be kept away from the convection zone, especially in the vicinity of the magnetic poles. To be sure, Toby and Michael have shown that a confined polar configuration can exist. However, in not only three-dimensional simulations of the kind carried out by Sacha Brun, Antoine Strugarek and Jean-Paul (Brun \& Zahn 2006; Strugarek, Brun \& Zahn 2011a,b), but also in the early axisymmetric two-dimensional simulations by Pascale Garaud (2002), who was able to achieve lower diffusion coefficients, a magnetic field initially concentrated well below the convection zone always diffused into the convection zone in the vicinity of the poles before a confining meridional tachocline flow was established. In part this was due to the neglect of downwelling from the convection zone, driven by the anisotropic turbulent stresses and advecting the magnetic field downwards against its natural tendency to diffuse outwards, as Pascale and her brother, Jean-Didier, (Garaud \& Garaud 2008) subsequently emphasized. The outcome was a magnetic torque transmitted between the convection and radiative zones which imparted the convection zone's differential rotation on the envelope beneath. However, there was reason for optimism because some of the two-dimensional computations exhibited transient confining behaviour, and indeed my conjecture was that because the magnetic field is strongest at the poles the strictly two-dimensional configuration is unstable: if the alignment of the magnetic and rotation axes were to be disturbed, the meridional tachocline flow would augment the perturbation and advect the magnetic axis equatorwards until it settled at the latitude of zero vertical shear (Gough 2012), a situation which cannot be modelled by the two-dimensional simulations. More about that later, for it is now not a necessary issue for demonstrating field confinement: recently Luis Acevedo-Arreguin, Pascale and Toby (2013) have analysed the balance of the diffusing agents much more carefully than had been carried out in the past, and have succeeded in computing a plausible two-dimensional representation of an axisymmetric tachocline. They established a parameter subspace in which confinement is possible, and in which the Sun lies, yet in which other, three-dimensional, simulations do not because they are dominated by viscosity. Of course the model is still very idealized, and there is evidently much more work to be done for a full understanding of the dynamics, but this valuable contribution possibly points the way to a viable theory.

This apparent success does not necessarily prove the story that is unfolding. Indeed, Jean-Paul has taken very seriously a conjecture by Emese Forgács-Dajka and Kristóf Petrovay $(2001,2002)$ that the penetration into the radiative envelope of the oscillating magnetic field presumed to have been generated by a dynamo in the convection zone is such as to hold the radiative envelope rigid. I had a stimulating time working with Jean-Paul on this idea during my final long-term encounter with him. Jean-Paul had kindly arranged that I visit him, now in Meudon, in the autumn of 2005, some four months after I was to have visited my scientific friends in Tokyo. Unfortunately, I suffered a severe constriction of my spinal chord, and 
was constrained to extend my stay in Japan for several months until the problem had been sorted out. Finally I had (extremely good) surgery, after which I flew to Boulder, where I was able to relax physically as the grateful guest of Jüri Toomre, and then straight to Paris, missing my homeland in order to minimize travelling. Jean-Paul and Suzy, very kindly as always, lent Rosanne and me one of their cars, so that Rosanne would not have to go to England to collect ours.

A criticism that could be levelled against Forgács-Dajka and Petrovay's calculation is that their generation of the oscillating magnetic field by the turbulent dynamo was unsheared by the differential rotation. Together, Jean-Paul and I studied a model in which an oscillating axisymmetric electric current partaking in the differential rotation generated a magnetic field in the convection zone, which diffused downward subject to a presumed uniform scalar turbulent diffusion coefficient into the much-less-diffusive radiative envelope. As in other studies, the angular velocity in the convection zone was prescribed to be consistent with helioseismology; then the response of an initially uniformly rotating radiative zone beneath was investigated. Jean-Paul carried out a numerical calculation, using a computer programme supplied by Antia (2002). It was necessarily viscously dominated, and appeared to lead to a smooth variation of angular velocity which was nearly uniform in the radiative envelope. I attacked the problem analytically, and was able to adopt realistic diffusion coefficients. The outcome was quite different. It involved a complicated evolving boundary layer, the details of which were not completely worked out. However, if the radiative zone were deemed to be rotating rigidly, the dominating oscillatory component of the Maxwell stress exerted in that zone was accompanied by a rectified term which varied in latitude in proportion to the differential rotation of the convection zone, demonstrating that uniform rotation, even when temporally averaged, cannot be the final outcome.

\section{Sector structure}

If the interior magnetic dipole is aligned with the latitude of zero vertical shear in the tachocline, as in Figure 3, one might expect an observable nonaxisymmetric consequence, rotating more-or-less at the speed of the radiative envelope, yet on average maintaining phase. The large-scale photospheric magnetic field distribution associated with coronal holes comes immediately to mind, but there appears to be no analysis over a sufficiently long time interval to establish whether phase is adequately maintained ${ }^{5}$. However, there is an interesting study by Leif Svalgaard and John Wilcox (1975) of the long-term variation of the geomagnetic field, which is believed to be intimately connected to the sector structure of the solar wind. Leif and John had plotted the polarity of the field against time, over a duration of nearly half a century, and two approximately periodic components were identified by eye. Emphasis was on one with a period of 26.84 days synodic, believed to be a signature of the solar dynamo operating in the equatorial regions of the

\footnotetext{
${ }^{5}$ Giuliana de Toma, personal communication.
} 

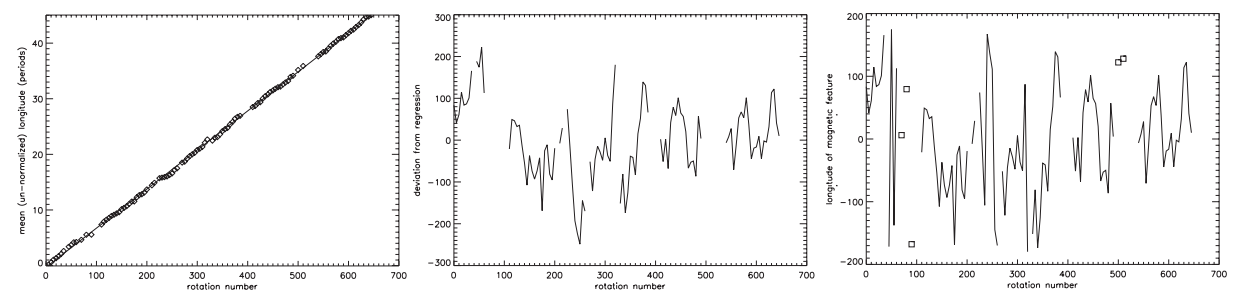

Fig. 4. Projected longitudes, in units of the fiducial rotation period of 26.84 days, of the centres of Svalgaard and Wilcox's (1975) secondary oscillatory geomagnetic field component.

convection zone. The other, less obvious, secondary component had a period of about 28.7 days synodic. Could that be the evidence sought for the global dipole field? In Figure 4 is plotted the "longitudes", after imaginary rigid projection onto the Sun, of the centres of the secondary component (in units of the fiducial 26.84-day period). There are gaps in the data, near sunspot minima when the field variation was too feeble to be identified, but each time when the signal reappeared it was almost in phase. The straight line in the figure was obtained by linear regression: its slope is 0.07025 periods/period, corresponding to a sidereal cyclic frequency of $434.6 \mathrm{nHz}$. That is just the rotation rate $\Omega_{0} / 2 \pi$ of the Sun's radiative interior (Fig. 1 ).

I had discussed with Jean-Paul the possibility of an investigation such as this when last we were together, taking time off from the first ISIMA programme at the University of California, Santa Cruz in the summer of 2010. We were spending a very pleasant long weekend with Suzy and Rosanne in the Napa Valley. At that time no scientific progress had yet been made: I had not yet become aware of Leif and John's geomagnetic study. And, besides, in such a wonderful wine-producing area we had more interesting activities to pursue. However, I'm sure that if JeanPaul were with us today he would find the result very interesting too. Whether or not he would have agreed with the proposed implications, he would certainly have shared with us his infectious enthusiasm. I, like all his friends, miss him sorely.

\section{References}

Antia, H.M., 2002, Numerical Methods for Scientists and Engineers, Birkh, ASIN: B01K03Q60O

Acevedo-Arreguin, L.A., Garaud, P., \& Wood, T.S., 2013, ApJ, 434, 720

Bondi, H., \& Lyttleton, R.A., 1948, Proc. Camb. Phil. Soc., 44, 345

Booker, J.R., \& Bretherton, F.P., 1967, JFM, 27, 513

Braithwaite, J., \& Spruit, H.C., 2004, Nature, 431, 819

Brown, T.M., Christensen-Dalsgaard, J., Dziembowski, W.A., et al., 1989, ApJ, 343, 526

Brun, A.S., \& Zahn, J-P., 2006, A\&A, 457, 665

Charbonneau, P., \& MacGregor, K.B., 1993, ApJ, 417, 762 
Diamond, P.H., Itoh, S-I., Itoh, K., \& Silvers, L.J., 2007, in The Solar Tachocline, ed. D.W. Hughes, R. Rosner \& N.O. Weiss, p. 213

Eliassen, A., \& Palm, E., 1960, Geof. Publ., 22, 3, 1

Elliott, J.R., 1997, A\&A, 327, 1222

Elliott, J.R., \& Gough, D.O., 1999, ApJ, 516, 475

Forgács-Dajka, E., \& Petrovay, K., 2001, Sol. Phys., 203, 395

Forgács-Dajka, E., \& Petrovay, K., 2002, A\&A, 389, 629

Fritts, D.C., Vadas, S.L., \& Andreassen, O., 1998, A\&A, 333, 343

Garaud, P., 2001, MNRAS, 324, 68

Garaud, P., 2002, MNRAS, 329, 1

Garaud, P., \& Garaud, J-D., 2008, MNRAS, 391, 1239

Goode, P.R., Dziembowski, W.A., Korzennik, S.G., \& Rhodes, E.J., 1991, ApJ, 367, 649

Gough, D.O., 1976, in Proc. Intl Colloq. Drops and Bubbles, ed. D.J. Collins, M.S. Plesset \& M.M. Saffren, vol. 1, US Gov. Printing Office 685-197/2

Gough, D.O., 1998, Space Sci. Rev., 85, 141

Gough, D.O., 2012, GAFD, 106, 429

Gough, D.O., \& McIntyre, M.E., 1998, Nature, 394, 755

Greenspan, H.G., 1969, The Theory of Rotating Fluids (Cambridge University Press)

Haynes, P.H., McIntyre, M.E., et al., 1991, J. Atmos. Sci., 48, 651

Holton, J.R., \& Lindzen, R.S., 1972, J. Atmos. Sci., 29, 1076

Howe, R., Christensen-Dalsgaard, J., Hill, H., Komm, R.W., Larsen, R.M., Schou, J., Thompson, M.J., \& Toomre, J., 2000, Science, 287, 2456

Kim, E-J., 2003, A\&A, 441, 763

Kim, E-J., \& MacGregor, K.B., 2003, ApJ, 588, 645

Kitchatinov, L.L., \& Rüdiger, G., 2006, A\&A, 435, 329

Kumar, P., Talon, S., \& Zahn, J-P., 1999, ApJ. 520, 859

MacGregor, K.B., \& Charbonneau, P., 1999, ApJ, 519, 911

McIntyre, M.E., 2003, in Stellar astrophysical fluid dynamics, ed. M.J. Thompson \& J. Christensen-Dalsgaard (Cambridge University Press), p. 111

Mestel, L., \& Weiss, N.O., 1987, MNRAS, 226, 123

Pedlosky, J., 1979, Geophysical Fluid Dynamics (Springer), New York

Plumb, R.A., \& McEwan, A.D., 1978, J. Atmos. Sci., 35, 1827

Rogers, T.M., 2011, ApJ, 733, 12

Rosner, R., \& Wiess, N.O., 1985, Nature, 317, 790

Rüdiger, G., \& Kitchatinov, L.L., 1997, AN, 5, 273

Sekii, T., 1991, PASJ, 43, 381

Spiegel, E.A., 1972, in Physics of the Solar System, ed. S.I. Rasool, NASA SP-300 (Washington, DC), p. 61

Spiegel, E.A., \& Zahn, J-P., 1992, A\&A, 265, 106

Strugarek, A., Brun, A.S., \& Zahn, J-P., 2011a, A\&A, 532, A34

Strugarek, A., Brun, A.S., \& Zahn, J-P., 2011b, AN, 332, 891

Svalgaard, L., \& Wilcox, J.M., 1975, Sol. Phys. 41, 461

Taylor, G.I., 1915, Phil. Trans. R. Soc. London, Ser. A, 215, 1 
Taylor, G.I., 1932, Proc. R. Soc. London, Ser. A, 135, 685

Thompson, M.J., Toomre, J., \& The GONG Dynamics Inversion Team, 1996, Science, 272,1300

Tobias, S.M., Diamond. P.H., \& Hughes, D.W., 2007, ApJ, 667, L113

Wood, T.S., \& McIntyre, M.E., 2011, JFM, 677, 445

Zahn, J.-P., 1974, Proc. IAU Symp., 59, 185

Zahn, J.-P., 1992, A\&A, 265, 115

Zahn, J.-P., 1994, A\&A, 288, 829

Zahn, J.-P., Talon, S., \& Matias, J., 1997, A\&A, 322, 320 